\title{
Orangutans modify facial displays depending on recipient attention
}

Bridget Waller, Cátia Correia Caeiro, Marina Davila-Ross

Primate facial expressions are widely accepted as underpinned by reflexive emotional processes and not under voluntary control. In contrast, other modes of primate communication, especially gestures, are widely accepted as underpinned by intentional, goal-driven cognitive processes. One reason for this distinction is that production of primate gestures is often sensitive to the attentional state of the recipient, a phenomenon used as one of the key behavioural criteria for identifying intentionality in signal production. The reasoning is that modifying/producing a signal when a potential recipient is looking could demonstrate that the sender intends to communicate. Here, we show for the first time that the production of a primate facial expression can also be sensitive to the attention of the play partner. Using the orangutan (Pongo pygmaeus) Facial Action Coding System (OrangFACS), we demonstrate that facial movements are more intense and more complex when recipient attention is directed towards the sender. Therefore, production of the playface is not an automated response to play (or simply a play behaviour itself) and is instead produced flexibly depending on the context. If sensitivity to attentional stance is a good indicator of intentionality, we must also conclude that the orangutan playface is intentionally produced. However, a number of alternative, lower level interpretations for flexible production of signals in response to the attention of another are discussed. As intentionality is a key feature of human language, claims of intentional communication in related primate species are powerful drivers in language evolution debates, and thus caution in identifying intentionality is important. 
2 Bridget M. Waller

3 Centre for Comparative and Evolutionary Psychology, Department of Psychology, University of

4 Portsmouth, UK

5

6 Cátia C. Caeiro

7 Centre for Comparative and Evolutionary Psychology, Department of Psychology, University of

8 Portsmouth, UK

9 Perception, Action and Cognition Research Group, School of Psychology, University of Lincoln, UK

10

11 Marina Davila-Ross

12 Centre for Comparative and Evolutionary Psychology, Department of Psychology, University of

13 Portsmouth, UK 
15 Nonhuman primate facial expressions (as well as their human counterparts) have long been considered

16 to be hard-wired, emotional displays (e.g. Darwin 1872). Facial expressions are often contrasted to

17 nonhuman primate (hereafter primate) gestures, which are not thought to be underpinned by automated,

18 emotional mechanisms, and instead widely believed to be intentional signals (Tomasello 2008). Such

19 distinctions between the different types of primate communication are used as crucial platforms to

20 develop and support theories of language evolution (see Slocombe, Waller et al. 2011). The

21 behavioural data supporting these conclusions, however, is incomplete in the sense that different

22 modalities are rarely examined within the same methodological framework, and instead each study

23 focuses on a specific modality in isolation (Slocombe, Waller et al. 2011). Moreover, the dichotomy

24 between emotionality and intentionality could be false, and communicative signals might not

25 necessarily be tied to one process or the other (Maiese 2014, Demuru, Ferrari et al. 2015).

Efforts to understand the evolution of human language, the production of which is highly

dependent on intentionality and related abilities such as theory of mind (Hockett 1960, Dennett 1989),

often search for evidence of intentionality in the communication of other animals in order to identify evolutionary antecedents (Liebal, Pika et al. 2004, Leavens, Russell et al. 2005, Genty, Breuer et al. 2009, Schel, Townsend et al. 2013). Definitions of intentionality are debated both within and between

31 groups of philosophers, psychologists, biologists and others, but broadly defined, intentionality refers

32 to acts and thoughts that are goal-directed, voluntary and purposeful (Grice 1957, Dennett 1983). The

33 conservative position is that animal communication is not intentional, unless systematic evidence 34 suggests otherwise.

Despite the obvious difficulty in determining when any animal behaviour is intended or not, researchers have attempted to use various observable behaviours to distinguish intentional communication from unintentional, automated communication. Leavens and colleagues (2005) 
39 of children: social use (production of the signal is sensitive to a social audience), gaze alternation (the

40 sender looks between the recipient and event/object), attention-getting behaviours (sender attempts to

41 attract recipient's attention), persistence (repeated use of signal), elaboration (increased intensity and

42 modification of signal to engage recipient) and sensitivity to attentional state (sender adjusts signals

43 depending on visual attention of recipient). These criteria for intentionality have become established in

44 the field of primate communication, but their application varies between studies. For example, some

45 studies use two or more of these criteria as sufficient evidence of intentionality (Schel, Townsend et al.

46 2013), whereas others use only one (Tempelmann and Liebal 2012). Some authors argue, however, that

47 multiple (if not all) criteria should be met (Genty, Breuer et al. 2009, Schel, Townsend et al. 2013).

48 Attentional stance sensitivity and social use were the most commonly used criteria for identification of

49 intentionality in a recent review of 24 published studies (18 of 24 studies used social use, and 16 of 24

50 used attentional stance: Liebal, Waller et al. 2014). The review found that two studies used attentional

51 stance sensitivity (Hobaiter and Byrne 2011, Hobaiter and Byrne 2011) and five used social use

52 (Tomasello, George et al. 1985, Liebal, Pika et al. 2004, Hobaiter and Byrne 2011, Hobaiter and Byrne

53 2011, Tempelmann and Liebal 2012) as sufficient criteria for the identification of intentionality in

54 primate's communication (as in, when only one of several criteria was necessary for intentionality to

55 be attributed).

56 Social use, therefore, is by far the most commonly applied behavioural criterion for

57 intentionality. Social use, however, has been highly criticised as a true marker of intentional production

58 of a signal, as one could argue that a social audience can trigger communicative behaviours through

59 several low-level mechanisms such as increased arousal, reflexive responses to the presence of another

60 and so on (Liebal, Waller et al. 2014). Sensitivity to attentional stance has been argued to be a more

61 resilient marker of intentional communication (Liebal, Waller et al. 2014) as communicating more/only

62 when the recipient is capable of receiving the message (i.e. visually attending) could be evidence that 
63 the sender has a goal-directed intention to communicate, and even an understanding of the visual

64 perspective of others. However, alternative explanations such as sensitivity to the face and eyes of

65 others, or learning that responses are only achieved when others are facing, are also highly possible.

66 Indeed, the senses and responses of receivers have long been known as important factors in shaping

67 and constraining the evolution and development of communicative signals (e.g. receiver psychology:

68 Guilford and Dawkins 1991).

Despite the widespread claims that primate facial expressions are used less intentionally than

70 primate gestures, several studies have found that facial expressions can be sensitive to the attentional

71 stance of others. As the primary focus of these studies was often gesture, however, the facial

72 expressions were broader movements sometimes referred to as facial gestures (e.g. head bob, head

73 shake: Liebal, Call et al. 2004) rather than prototypical facial expressions involving facial muscles.

74 Some studies have also examined responses to a human demonstrator rather than a conspecific in

75 spontaneous social interaction (Poss, Kuhar et al. 2006, Leavens, Russell et al. 2010). Liebal, Pika et

76 al. (2004), however, included prototypical facial expressions in their study of siamang social

77 communication, and found that the vast majority of grins and mouth-opens were produced only in the

78 presence of an attending recipient. Scheider, Waller et al. (in prep) have also found that hylobatid facial

79 expressions are longer when facing a conspecific in a variety of social interactions, and Demuru,

80 Ferrari et al. (2015) found that bonobos produce play facial expressions more often when their play

81 partner can see them. Moreover, sensitivity to attentional stance in facial signalling may not be

82 restricted to the primate order, as Horowitz (2009) found some evidence that domestic dogs produce

83 facial play signals more often when in the presence of an attentive play partner than an inattentive

84 partner. Demuru, Ferrari et al. (2015) argue that such data demonstrate the combined emotional and

85 intentional properties of play facial expressions, building on the neuro-anatomical thesis that emotional 
86 and intentional systems both underpin facial expressions (Sherwood, Holloway et al. 2004) and are 87 intimately intertwined (Cattaneo and Pavesi 2014).

The focus of the current study, therefore, was to test whether one of the most commonly applied criteria (sensitivity to attentional stance) claimed to demonstrate intentionality can be applied rigorously to the production of a prototypical orangutan facial expression. The relaxed open mouth

91 display (playface, Fig. 1) is a facial expression ubiquitous in the primate order and almost exclusively restricted to play contexts (van Hooff 1973, Parr, Cohen et al. 2005). Similar facial expressions are also

93 found in other mammals (see Waller and Micheletta 2013) and so it seems highly preserved from a

94 phylogenetic perspective. The prototypical form of the playface is similar across primate species,

95 involving an open mouth and exposure of the lower and (in some species) upper teeth (Preuschoft

96 1992, Palagi, Antonacci et al. 2007, Davila Ross, Menzler et al. 2008, Palagi 2008, Waller and Cherry

97 2012). The playface has been proposed as a homologue of the human laughter display (van Hooff

98 1972) and as a ritualised form of mock biting during play, as if to demonstrate that play is only play

99 (Bolwig 1964, Pellis and Pellis 1996).

The characteristics of the playface therefore, are not suggestive of complex underlying cognition, such as developmental sensitivity, flexibility of use, referentiality or intentionality (for a

102 review of the relevance of these features see Liebal, Waller et al. 2014). However, even if there is a

103 relatively fixed component to facial expression production, it could quite conceivably still be

104 underpinned by both emotional and intentional processes. For example, catarrhine primates have

105 control of the facial muscles through direct cortical connections, suggesting an element of voluntary

106 control (Sherwood 2005). Thus, it is possible that primates can modify the playface in response to their 107 audience.

108 The goal of this study was to use OrangFACS (Caeiro, Waller et al. 2013) to determine whether 109 orangutans (Pongo pygmaeus) modify their facial signals depending on the attentional stance of 
110 another individual (the recipient) during spontaneous play interactions, and thus meet one of the

111 established criteria for intentionality. We also recorded additional variables to control for potential

112 confounds of more intense play when face-to-face, and responses to the recipient's facial expression.

113 We recorded playfaces during social play, and examined the influence of 1) the recipients orientation

114 towards the sender, 2) the recipient's facial expression, and 3) intensity of play, on the complexity

115 (number of facial muscle components) and intensity (extent of mouth opening) of the playface.

116 


\section{METHODS}

\section{Study area and subjects}

120 The spontaneous dyadic play of 20 orangutans was observed in total. Nineteen individuals

121 (seven females, twelve males; 3-12 years old) featured as focal individuals in the analysis (one was 122 included in the analysis as a recipient only as the roles of focal and recipient were randomly assigned 123 when each play bout was coded, and he never produced facial expressions when allocated the focal 124 role). Twelve individuals were housed at the nursery of the Sepilok Orangutan Rehabilitation Centre 125 (SORC), Malaysia. Inside enclosures consisted of cages where the orangutans stayed overnight either 126 individually or in pairs, and in larger groups during the day. They were taken outdoors (outside their 127 cages) for several hours in the morning and afternoon as part of their training programme where they 128 were filmed during spontaneous play. The remaining eight individuals were semi-free ranging as they

129 had been previously released by SORC into the Kabili Sepilok Forest Reserve. They lived in this forest 130 area during day and night, and were filmed during spontaneous play. Feeding took place three times 131 per day. The nursery-housed orangutans were fed inside their cages. The released orangutans obtained 132 the food from feeding platforms in the forest (provided by SORC), but they were also showing natural 133 foraging behaviours. There was no interaction between the individuals from SORC and the Forest 134 Reserve during the data collection period.

136 Video data collection

137 A total of 12 hours of spontaneous play behavior was extracted from 39 hours of ad libitum 138 (Altmann, 1974) social interaction footage (mean duration $=37 \mathrm{~min} \pm 20.38$ SD per individual). 139 Recordings were obtained outdoors between $8 \mathrm{am}$ and $12 \mathrm{pm}$ and between $2 \mathrm{pm}$ and $5 \mathrm{pm}$ from August to 140 October in 2005. Recordings were taken from no more than $10 \mathrm{~m}$ away from the play dyad by a 
141 handheld video camera, with both animals kept in view as much as possible. Play was identified based 142 on specific play actions (e.g., wrestling, hitting, grappling: Davila Ross et al. 2008) and only dyadic 143 play that occurred during the footage was extracted (solitary or triadic play was ignored to allow 144 analyses to control for identity of senders and receivers) for the purpose of this study. Research 145 permission was provided by Sabah Wildlife Department and Economic Planning Unit, Malaysia.

146

147

148

149

150

151

152 153

154 155 156

\section{Behavioural coding}

The video footage was then coded frame-by-frame (25 FPS) using Adobe Premiere Pro CS4 v.4 and Mangold Interact software. In each play dyad, one of the individuals was randomly chosen as the focal individual. All open mouth facial expressions (OMF) were identified using a broad, inclusive operational definition based on OrangFACS (all occurrences where the mouth was opened by AU26 (jaw drop) or AU27 (mouth stretch) to avoid a priori assumptions about the form of play facial expressions). Any OMFs with poor visibility, where the onset was not visible, or where there was physical biting were discarded. OMFs were treated as separate events if the mouth was fully closed for at least $2 \mathrm{~s}$ between movements.

The following binomial factors were coded for every OMF, and when any behaviour was not clearly discernable it was marked as unscorable:

1) Facial orientation: whether the individuals were facing each other and had an unobstructed view of each other's face within an angle of 45 degrees of head rotation (Fig. 2: face to face, FTF; or not face to face, Not FTF). Each play session was split into multiple periods of FTF and Not FTF play (so each OMF could be classed as FTF or not).

2) Recipient facial expression: whether the recipient individual displayed an OMF at any point during the duration of the focal OMF (OMFR or nOMFR). 
166

167

168

169

170

171

172

173

174

175

176

177

178

179

180

181

182

183

184

185

186

187

188 Reliability coding

3) Play intensity: the speed, strength and degree of physical contact of play behaviour between focal and recipient individuals during an OMF (low or high). Play bouts including resting, temporary breaks from play and slow grappling were classed as low intensity play. Play bouts containing chasing, gnawing, grappling, hitting and wrestling were classed as high intensity.

\section{Facial movement coding}

FACS (Facial Action Coding Systems) are useful tools to quantify subtle changes in primate

facial signals. The first FACS was developed as an anatomically based observational tool for the measurement of facial movement in humans (Ekman and Friesen 1978), and has since been modified for use with other animals: chimpanzees (Vick, Waller et al. 2007), rhesus macaques (Parr, Waller et al. 2010), gibbons (Waller, Lembeck et al. 2012), orangutans (Caeiro, Waller et al. 2013) and domestic dogs (Waller, Peirce et al. 2013). Individual facial muscle movements can be identified and quantified as Action Units (AUs), which allows an objective assessment of morphological changes in facial expressions without the need for a priori emotional labels (Waller and Smith Pasqualini 2013). Here,

OrangFACS (Orangutan Facial Action Coding System: Caeiro, Waller et al. 2013) was used to identify the facial movements produced during each OMF. A certified coder (CC) coded whether any of the following action units were present during the OMF using one/zero sampling (Altmann 1974): brow lowerer (AU4), cheek raiser (AU6), upper lip raiser (AU10), lip corner puller (AU12), lower lip depressor (AU16), jaw drop (AU26) or mouth stretch (AU27). These AUs were chosen as the full range of facial movements likely in an OMF, and all can be present simultaneously in the face with the exception of AU26 and AU27 (which are mutually exclusive). 
To test for data coding consistency, 30 clips of play were extracted at random from the footage

190 and coded (blindly to the study goal) at both the beginning and end of the project (one year apart).

191 Intra-reliability was measured using Cohen's Kappa (Cohen 1960). Good agreement was reached for

192 FTF vs. Not FTF $(K=0.66, P<0.0005)$ and non-focal OMF $(K=0.77, P<0.0005)$. Wexler's index

193 (Ekman, Friesen et al. 2002) was used for AUs, and led to a value of 0.87, which is considered

194 excellent agreement. The inter-observer reliability for play intensity had been assessed previously $(K=$ $1950.84, P<0.0005)$, and can be considered very good agreement.

Statistical analysis

Generalized linear mixed models (GLMM) were used to analyse our nested data, with defined linear hierarchical groups. The GLMM analysis allowed us to include random factors to control for the fact that: 1) the data set contained missing values (for some observations we could not code for all the

201 factors), 2) individuals appeared a different number of times as both focals and non-focals, 3) more than one OMF was collected from each play bout and/or from the same individual, and 4) not all individuals played together (since the play was spontaneous). We controlled for multiple observations of the same individuals from the same group by adding the identity of the individuals involved in the interaction nested within groups as a random factor and also added a third random factor to control for repeated dyad composition, thus avoiding pseudoreplication (Machlis, Dodd et al. 1985, Pinheiro and Bates 2009, Waller, Warmelink et al. 2013). The function vif.mer (Frank, 2011) was used to calculate collinearity between the factors. To compute the models, the glmer function from the lme4 package was used (Bates, Maechler et al. 2013). The GLMM were fit by maximum likelihood (ML) with

210 Laplace approximation. Instead of testing for the null-hypothesis to choose our factors, we used an 211 information-theoretic approach (Burnham and Anderson 2002). We computed ANOVAs and used a 212 combined backward and forward stepwise method, based on Akaike Information Criterion (AIC) to 
213 compare models and choose the best one (i.e. the model with the lowest AIC value: Burnham and

214 Anderson 2002, Field, Miles et al. 2012). Significance of factors within models was assessed using p-

215 values, which explains the impact of factors on the outcome variable as compared to each other. All the 216 statistical analyses were computed in R 3.1.1 (R Core Team, 2014).

218 RESULTS

A total of 247 OMFs (see Fig. 1 for an example) were analysed from 19 of the 20 individuals in our sample (mean OMF number \pm SD: $13 \pm 5.97$ per individual, see Table 1), during 121 play bouts 221 (mean bout duration \pm SD: $309.48 \mathrm{~s} \pm 482.43$ ). The remaining individual only ever featured as a 222 recipient and so was not included as a focal subject in the analysis. OMF duration ranged from $0.08 \mathrm{~s}$ 223 and 10.56s overall, and there was a significant difference in the duration of OMF in FTF (mean 224 duration \pm SD: $1.64 \mathrm{~s} \pm 1.19$ ) and Not FTF (mean duration \pm SD: $1.09 \mathrm{~s} \pm 0.71$ ) conditions (Wilcoxon 225 signed ranks test: $T=-2.20, N=19, P<0.05$ ). Note that more OMFs were observed than coded (Table 226 1), as visibility was not always good enough for FACS coding (GLMM analyses are robust and 227 suitable for datasets with missing data).

228 229 230 231 232 233 234 235 236

As the goal was to compare features of the OMF produced during FTF and Not FTF play, we controlled for the fact that OMF durations differed between conditions (and so the number of AUs produced could differ as a function of time rather than condition if they accumulate over time). We compared the onset latencies of AUs in FTF and Not FTF play in a random subset sample using Wilcoxon matched-pairs signed-rank test. We found no significant difference between the mean latency of AUs onset in FTF (mean latency \pm SD: $0.12 \mathrm{~s} \pm 0.13$ ) and Not FTF (mean latency $\pm \mathrm{SD}$ : $0.14 \mathrm{~s} \pm 0.21$ ) conditions (Wilcoxon signed ranks test: $T=0.00, N=6, P=1.000$ ). Therefore, as the start time of all AUs within each OMF is approximately the same time in both conditions (almost 
immediately at the onset of the expression), the length of the OMF cannot be a factor influencing the number of cumulated AUs, and AUs do not accumulate over time.

\section{Complexity of OMF as a function of recipient attention}

To investigate whether complexity of OMF (defined as the total number of individual AUs) varied depending on facial orientation, recipient facial expression and play intensity, we calculated GLMM with Poisson error distribution and log function. The total number of AUs in each OMF was used as a response factor and the identity of focal and recipient individuals as well as the play bout number were entered as random factors. Facial orientation (FTF vs Not FTF), recipient facial expression (OMFR vs nOMFR) and play intensity (low vs high) were entered as fixed factors. There was no overdispersion in the data set or collinearity in the factors.

The model that best fit the data was the full model, containing facial orientation, recipient facial expression and play intensity (see Table 2). The full model was compared to the null model, showing a highly significant difference: ANOVA: $F_{3}=417.26, P<0.001$. Removal of any of the three factors from the full model resulted in a significant change in the model's AIC, since all the factors were strongly influencing the facial movement complexity of the focal individual during play behaviour (best model AIC: 454.4 vs model without facial orientation AIC: 459.57 , ANOVA: $F_{1}=7.184, P<$ 0.001, vs model without recipient facial expression AIC: 812.6 , ANOVA: $\mathrm{F}_{1}=360.25, P<0.001$ and vs model without play intensity AIC: 476.15, ANOVA: $\mathrm{F}_{1}=23.767, P<0.001$, Fig. 3 ).

In the full model, facial orientation had a significant positive effect as a fixed factor $(P<0.01)$ : OMF produced when the recipient was facing the sender contained a greater number of AUs (mean number of $\mathrm{AUs} \pm \mathrm{SD}$ in FTF: $3.29 \pm 0.11$; mean number of AUs $\pm \mathrm{SD}$ in Not FTF: $2.54 \pm 0.11$ ). Facial expression of the recipient was not significant $(P=0.06)$, but as the AIC was lowered significantly when this was taken out of the model, it had a weak positive effect on the model: OMF produced when 
261 the recipient also produced an OMF contained a greater number of AUs (mean number of AUs \pm SD in

262 OMFR: $3.35 \pm 0.14$; mean number of AUs \pm SD in nOMFR: $2.60 \pm 0.17$ ). Play intensity was also not

263 significant as a fixed factor $(P=0.74)$. OMF produced when the play intensity was high or low

264 contained a similar number of AUs (mean number of AUs \pm SD in high play intensity: 2.97 \pm 1.19 ;

265 mean number of AUs \pm SD in low play intensity: $3.04 \pm 1.42$ ), but did significantly improve the model

266 fit (although in a positive direction, so low intensity play increased the likelihood of more AUs in the

267 OMF). Each of the factors alone represented a significantly better fit when compared to the null model

268 (facial orientation vs null model ANOVA: $F_{1}=10.969, P<0.001$; recipient facial expression vs null

269 model ANOVA: $F_{1}=385.49, P<0.001$; play intensity vs null model ANOVA: $\left.F_{1}=46.908, P<0.001\right)$

270 and thus had some impact on complexity of OMFs.

271

272

Intensity of OMF as a function of recipient attention

274

275

276

277

278

279

To test whether intensity of OMF varied (whether it contained AU26, a jaw drop, or AU27, the

stronger mouth stretch movement) depending on facial orientation, recipient facial expression and play intensity, we calculated GLMM with binomial error distribution and logit link function. AU26 versus AU27 was imputed as the binary response factor. The identity of focal and recipient and the play bout number were added to the model as random factors. The model was slightly overdispersed (i.e. more variance than expected by the standard model), so we added an OMF-level random factor (1|OMF), where OMF is a vector from 1 to the total number of observations (247) (Bolker, 2008).

The full model retaining all factors provided the best fit for the data (see Table 2). The full model had the lowest AIC (148.1) and removal of any of the factors resulted in a significant change to the model and when compared to the null model (facial orientation vs null model ANOVA $F_{1}$ $=17.279, P<0.001$; recipient facial expression vs null model ANOVA: $F_{1}=138.01, P<0.001$; play intensity vs null model ANOVA: $\left.F_{1}=16.374, P<0.001\right)$. When comparing the full best model to the 
285 null model, the result was also highly significant (full model vs null model ANOVA $F_{3}=156.77, P<$ $2860.001)$.

287 Within the fixed factors of the best model, facial orientation was the only significant factor $(P<$ 288 0.05), being more associated with the stronger AU27 movement than AU26. However, when

289 comparing models, recipient facial expression also had a strong (positive) significant influence (best 290 model AIC: 148.1 vs model without facial orientation AIC: 157.5 , ANOVA: $F_{1}=11.326, P<0.001$ 291 and vs model without recipient facial expression AIC: 270.3 , ANOVA: $F_{1}=124.16, P<0.001$ ), and 292 play intensity had a weak negative influence (best model AIC: 148.1 vs model without play intensity 293 AIC: 151.5 , ANOVA: $F_{1}=5.352, P<0.05$ ) on the display of AU26 versus AU27. As low intensity 294 was set as the baseline, a negative influence indicates that more intense play increased the likelihood of 295 AU27 vs AU26. Therefore, playfaces were more likely to contain the more intense AU27 when the 296 recipient was facing the sender (FTF), when the recipient produced an OMF, and when play was more 297 intense (see Fig. 4).

298

299 


\section{DISCUSSION}

302

303

304

305

306

307

308

309

310

In the current study, orangutans were sensitive to the visual attention of their social partner when producing facial expressions. During social play, if the focal individual was facing their play partner with unobstructed visual access between the two individuals, open-mouth expressions (playfaces) were more intense and contained more component movements. Although previous studies have interpreted similar findings as evidence of intentional communication, we have a more cautious interpretation. These findings may not demonstrate intentionality beyond doubt, but do show that production of the playface is not an automated response to play (or simply a play behaviour itself) and instead is highly flexible and dependent on subtle characteristics of the social context.

Modified and increased production of visual signals as a function of the recipient's attentional stance has been used as sufficient criteria for intentionality in previous studies of primate gesture (Hobaiter and Byrne 2011, Hobaiter and Byrne 2011), and as additional criteria in many other studies (e.g. Liebal, Call et al. 2004, Poss, Kuhar et al. 2006, Anderson, Kuwahata et al. 2007, Genty and Byrne 2010, Leavens, Russell et al. 2010). Sensitivity to attentional stance has been used as evidence for intentionality in signal production in this way, as it could demonstrate that the sender is intending to communicate (and is thus only communicating when the audience is receptive to the signal). If we accept this logic, and thus accept that this behavioural marker is a sufficient demonstration of intentional production, we must conclude that, like primate gestures, orangutan playfaces are produced intentionally.

It is possible, however, that sensitivity to attentional stance when producing signals demonstrates a degree of flexibility of production (which demonstrates it is not fully involuntary, reflexive or automatic) without the need to attribute goal-directed, purposeful communication. The decision to produce a playface may be voluntary in the same sense that the decision to bite, eat or run 
325 may be voluntary in some way - the animal is capable of inhibiting or increasing the behaviour if

326 conditions suggest such an approach might be sensible. Although part of the continuum of

327 intentionality, this is not the same as goal-directed, purposeful intention to communicate to another.

328 There are also other explanations that could be considered. First, face-to-face play could simply

329 be more arousing and stimulate the production of more play (of which the playface is a component).

330 Play intensity did have a weak positive impact on the intensity of the playface in our model, but also a

331 negative impact on the complexity of the playface. Therefore, play intensity does seem to be one

332 contributory factor (on intensity at least), but is not strong and does not influence the composition of

333 the signal. Thus, increased playface production during face-to-face play is not simply a function of

334 face-to-face play being more intense. Second, playfaces might be stronger during visual contact with

335 the play partner due to reflexive mimicry of the partner's facial expression (rapid facial mimicry: e.g.

336 Davila Ross, Menzler et al. 2008). The playfaces were more intense and more complex when the

337 recipient was also producing a similar facial expression, so mimicry could play a part, but as facial

338 orientation was also a strong factor in both models, mimicry cannot be the only explanation (although

339 may play a role). Third, primates may be responsive to the face of their play partner during play as the

340 face is a powerful stimulus for social primates and many species exhibit highly sophisticated facial

341 processing skills (Parr, 2011). Although such abilities may be a stepping-stone towards (and necessary

342 for) intentional communication, they might also be potential explanations for sensitivity to attentional

343 stance in and of themselves. Primate individuals respond to the faces of others during social interaction

344 on a regular basis, and so the face may act as a cue stimulating a response appropriate to the context.

345 For example, primates respond to threat faces with submissive expressions or counter threats, and

346 respond to subtle facial cues such as staring with overt behavioural responses (Yamagiwa, 1992). Such

347 a response would not necessarily require intentionality. 
Therefore, a number of alternative and additional explanations for sensitivity to visual attention

349 in communication are plausible, and we should be cautious when concluding that complex intentional

350 production has been demonstrated. Similar data, however, have been used to support the view that

351 primates are capable of intentional production of gestures to achieve strategic social goals. The

352 different research traditions underlying the study of facial expressions and gestures (Slocombe, Waller

353 et al. 2011, Liebal, Waller et al. 2014) may explain why different conclusions are being made in

354 different fields. Primate gesture is often proposed as a potential precursor to human language (a debate

355 which relies heavily on the data alluding to intentionality: Slocombe, Waller et al. 2011). The vast

356 majority of previous studies investigating intentionality in primate communication focus on gestures

357 (as opposed to vocalisations or facial expressions): all of the 24 studies reviewed in Liebal et al (2013)

358 examined gesture, nine examined vocalisation, and seven examined facial expression. These data are

359 consequently contributing to a body of work being cited as solid evidence that some species of

360 primates can communicate in a flexible, goal-orientated, and intentional fashion, particularly with

361 gestures. Furthermore, such data are being used as a crucial platform for the investigation of other

362 language-like characteristics (e.g. Genty and Zuberbuhler 2014, Hobaiter and Byrne 2014). Thus, even

363 if the gold standard is to use multiple criteria for the identification of intentionality, the implications of

364 using only one or few of the most commonly applied criteria (which is the status quo) needs to be

365 addressed.

366 Here, we demonstrated that production of orangutan facial expressions can be modified in

367 response to the presence and visual attention of another. Such modification may not be evidence of

368 intentional production, but it is nevertheless evidence of complexity within a communicative system

369 that has been hitherto overlooked. The difference in intensity and complexity of the playface between

370 facing and not facing conditions was only in degree, but extensive work on nonverbal behaviour in

371 humans and other animals suggests that even rapid and subtle cues can have an impact on social 
372 interaction. Further research is important to determine whether this sensitivity to attentional stance

373 does indeed have an important impact on consequent social interactions.

374 Facial expressions (in humans as well as other animals) have long been seen as rather fixed,

375 biologically based expressions of emotion, reflecting the internal state of the sender, a theoretical

376 stance reinforced since Darwin (1872). This may be true in some senses, but it is important that this

377 assumption does not influence the generation and interpretation of data a priori. Also, in the absence of

378 physiological data it may be just as difficult, and not necessarily more conservative, to conclude that a

379 signal is emotional rather than intentional (Waller and Micheletta 2013, Liebal, Waller et al. 2014) and

380 so concluding that a signal is emotional may not necessarily be a more conservative explanation.

381 Ultimately, a multimodal approach to primate communication might help overcome some of the

382 constraints surrounding the study of primate communication by promoting behavioural criteria for the

383 detection of intentionality to be used cautiously and consistently across species and communicative

384 modalities. Importantly, sensitivity to attentional stance may not demonstrate intentionality akin to that

385 used in human language, and so perhaps the significance of this trait in primate communication needs

386 to be reconsidered. Yet claims of intentional communication in related primate species abound in

387 language evolution debates, and thus caution in identifying intentionality is crucial.

\section{ACKNOWLEDGMENTS}

390 Recordings were obtained at Sepilok Orangutan Rehabilitation Centre and Kabili Sepilok Forest

391 Reserve, following the approval for research by Sabah Wildlife Department and Economic Planning

392 Unit, Malaysia. Thanks go to E. Zimmermann for contributions to the field research, to E. Bosi, H.

393 Bernard, and S. Alsisto for logistic help and to M. Wessels for assisting with the data collection. We

394 thank Jerome Micheletta, Juliane Kaminski and Jamie Whitehouse for comments on the manuscript. 
REFERENCES

398

FIGURES LEGENDS

399

400

401

Figure 1. Example of open mouth facial expression (playface) from (Davila Ross, Menzler et al. 2008).

402

403 Figure 2. Examples of face to face (FTF) and not face to face (Not FTF) conditions. To be classed as

404 FTF the two individuals had to have an unobstructed view of each other's face within an angle of 45

405 degrees of head rotation.

406

407 Figure 3. The number of OMF (open mouth facial expressions) containing different numbers of AUs

408 (action units) as (a) a function of facial orientation (FTF = face to face, Not FTF - not face to face), (b)

409 as a function of the facial expression of the recipient (With OMF $=$ recipient has OMF, no OMF -

410 recipient does not have OMF), and (c) as a function of play intensity (low and high).

411

412

413 Figure 4. Proportion of OMF (open mouth faces) with AU26 (jaw drop) and the more intense AU27

414 (mouth stretch) as (a) a function of facial orientation (FTF = face to face, Not FTF - not face to face),

415 as (b) a function of the facial expression of the recipient (OMFR = recipient has OMF, nOMFR -

416 recipient does not have OMF), and (c) as a function of play intensity (low and high). 


\section{1}

Image of orangutan play face

Example of open mouth facial expression (playface) from ( Davila Ross et al. 2008 ) . 


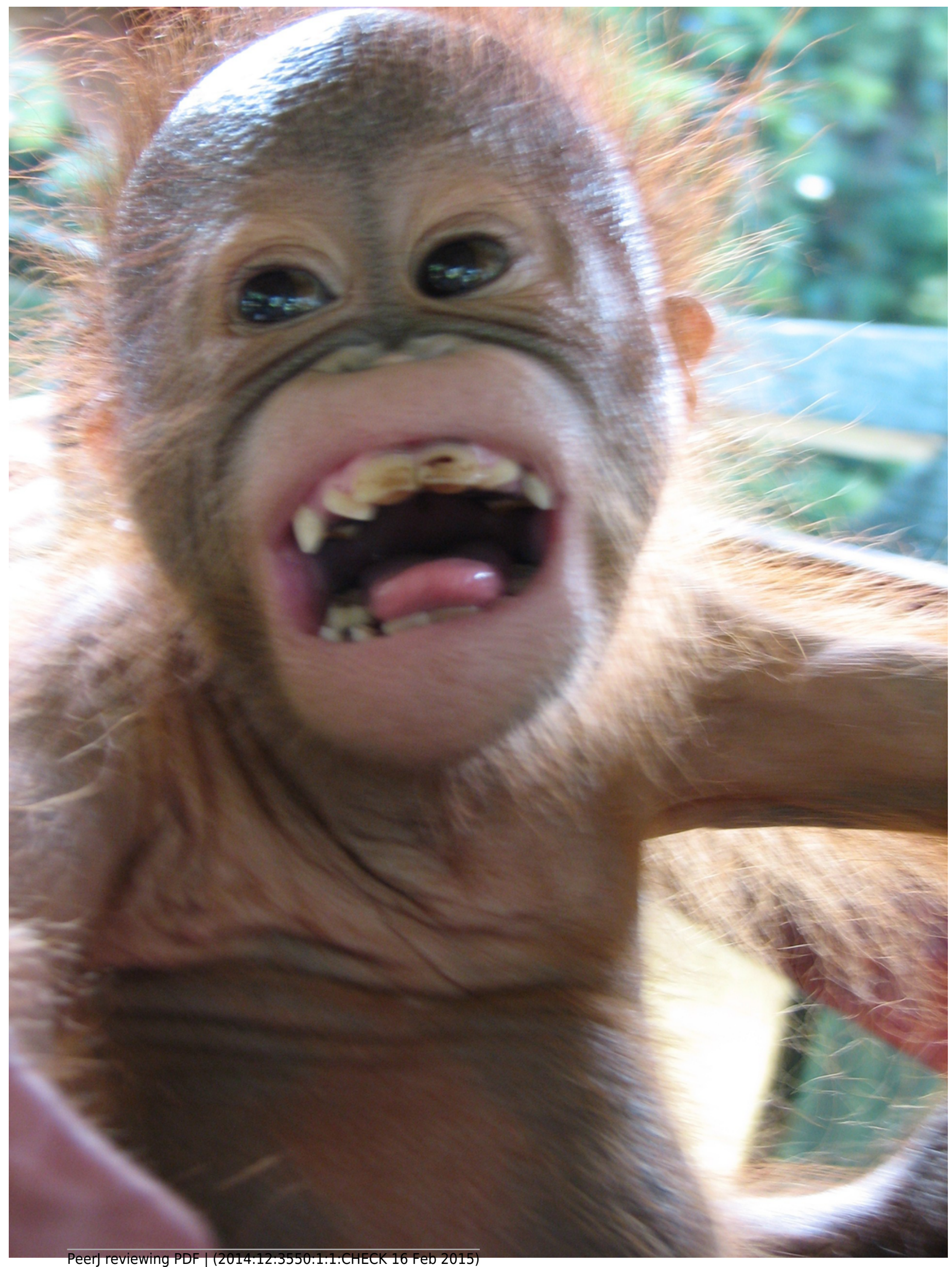


2

To be classed as FTF the two individuals had to have an unobstructed view of each other's face within an angle of 45 degrees of head rotation. 


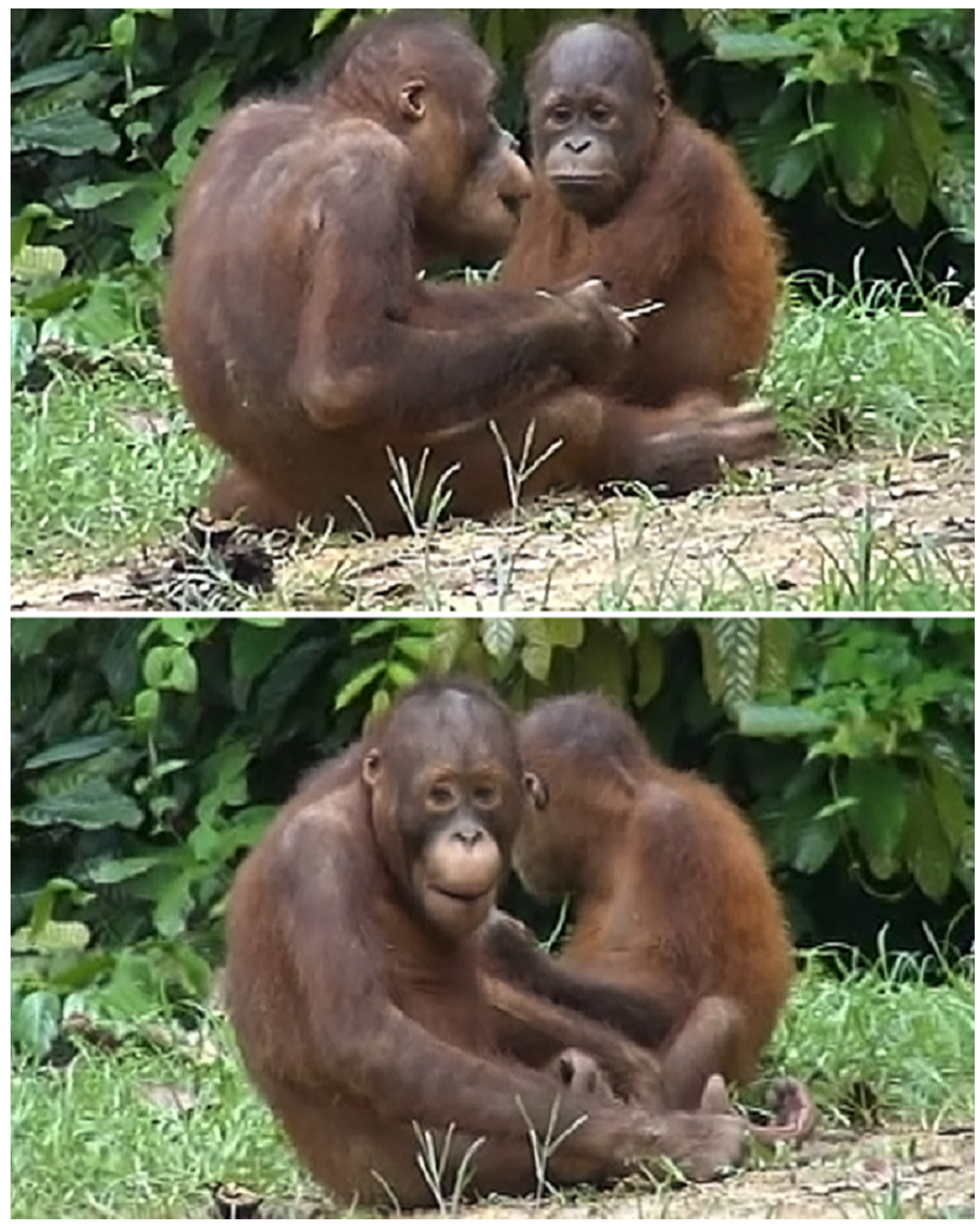




\section{3}

Figure showing number of OMF in different conditions.

The number of OMF (open mouth facial expressions) containing different numbers of AUs (action units) as (a) a function of facial orientation (FTF = face to face, Not FTF - not face to face), (b) as a function of the facial expression of the recipient (With OMF = recipient has OMF, no OMF - recipient does not have OMF), and (c) as a function of play intensity (low and high). 

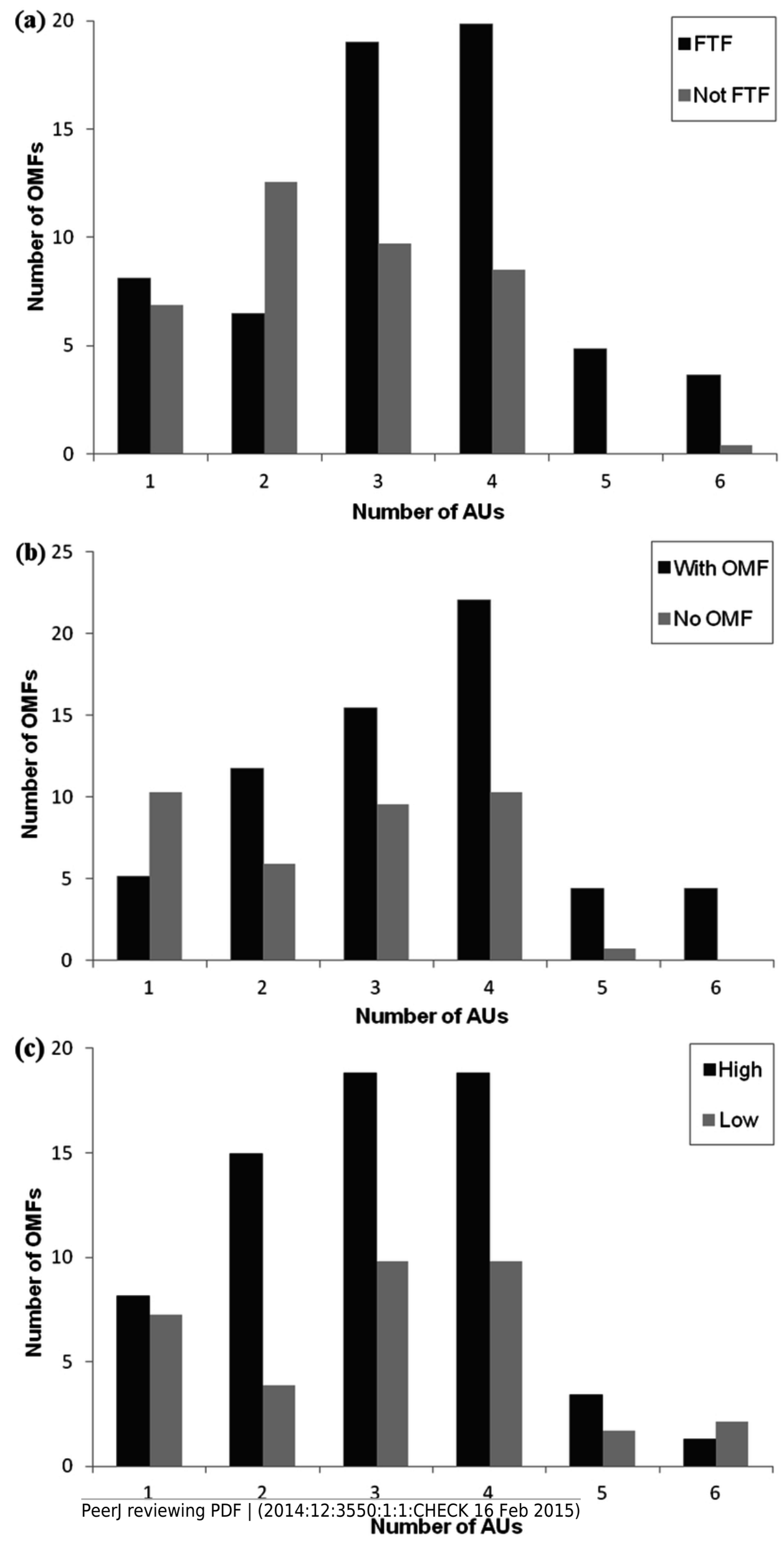


\section{4}

Figure showing proportion of OMF with different intensities in different conditions

Proportion of OMF (open mouth faces) with AU26 (jaw drop) and the more intense AU27 (mouth stretch) as (a) a function of facial orientation (FTF = face to face, Not FTF - not face to face), as (b) a function of the facial expression of the recipient (OMFR = recipient has OMF, nOMFR - recipient does not have OMF), and (c) as a function of play intensity (low and high). 
AU26

AU27
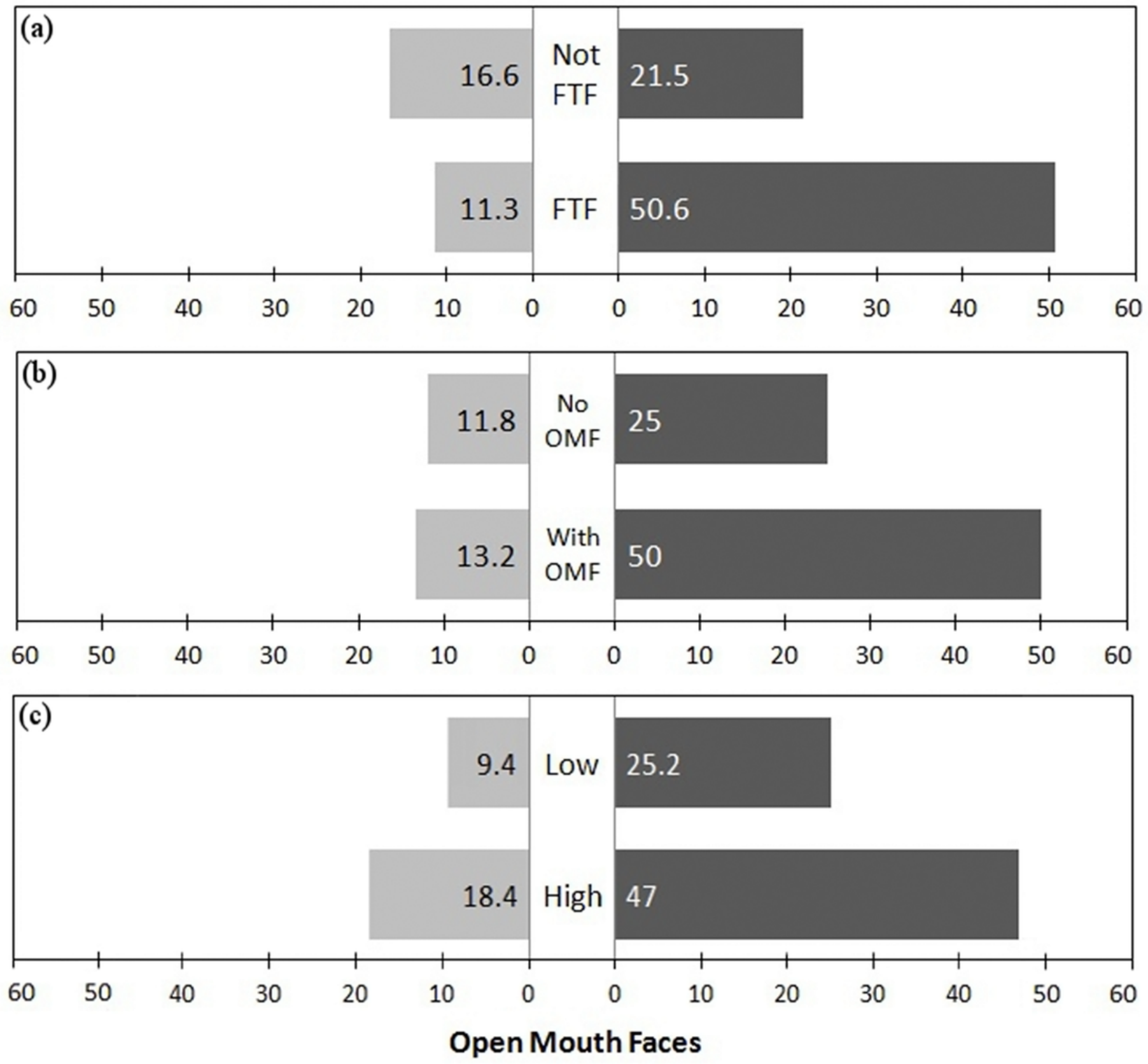


\section{Table $\mathbf{1}$ (on next page)}

Table of OMF data per individual 
2 Table 1. Distribution of OMF events per focal individual in each factor: Face to face, recipient OMF

3 and play intensity. OMFs were discarded whenever FTF or not FTF could not be scored, while

4 unscorable OMFs in recipient OMF and play intensity were maintained and coded as missing data

5 points.

6

\begin{tabular}{|c|c|c|c|c|c|c|c|c|}
\hline \multirow{2}{*}{$\begin{array}{l}\text { Focal } \\
\text { individual }\end{array}$} & \multirow[t]{2}{*}{$\begin{array}{c}\text { Group }(\mathrm{N}= \\
\text { nursery, } \mathrm{SF} \\
=\text { semi-free } \\
\text { ranging) }\end{array}$} & \multirow[t]{2}{*}{$\begin{array}{c}\text { Age } \\
\text { (years) }\end{array}$} & \multicolumn{2}{|c|}{ Face to face } & \multicolumn{2}{|c|}{$\begin{array}{l}\text { Recipient } \\
\text { OMF }\end{array}$} & \multicolumn{2}{|c|}{$\begin{array}{c}\text { Play } \\
\text { intensity }\end{array}$} \\
\hline & & & Yes & No & Yes & No & $\begin{array}{l}\text { Lo } \\
\mathrm{W}\end{array}$ & $\begin{array}{c}\mathrm{Hig} \\
\mathrm{h}\end{array}$ \\
\hline Anekara & $\mathrm{N}$ & 2 & 6 & 8 & 3 & 4 & 7 & 7 \\
\hline Ankong & SF & 4 & 9 & 8 & 5 & 3 & 1 & 12 \\
\hline Annelisa & $\mathrm{N}$ & 5 & 4 & 3 & 0 & 2 & 5 & 2 \\
\hline Anpal & $\mathrm{SF}$ & 3 & 13 & 4 & 4 & 5 & 10 & 7 \\
\hline Boy & SF & 7 & 8 & 4 & 4 & 4 & 5 & 7 \\
\hline Brock & $\mathrm{N}$ & 3 & 5 & 2 & 3 & 1 & 3 & 4 \\
\hline Dogi & $\mathrm{SF}$ & 7 & 14 & 3 & 7 & 0 & 5 & 10 \\
\hline Kam Chong & $\mathrm{SF}$ & 8 & 7 & 11 & 3 & 5 & 0 & 17 \\
\hline Kimbol & $\mathrm{N}$ & 3 & 10 & 8 & 5 & 5 & 7 & 11 \\
\hline Mico & SF & 5 & 23 & 5 & 12 & 8 & 12 & 12 \\
\hline Miskam & $\mathrm{SF}$ & 12 & 13 & 4 & 8 & 3 & 5 & 12 \\
\hline Naru & $\mathrm{N}$ & 2 & 9 & 7 & 4 & 2 & 6 & 10 \\
\hline Nonong & $\mathrm{N}$ & 3 & 3 & 2 & 1 & 1 & 1 & 4 \\
\hline Oscar & $\mathrm{SF}$ & 6 & 7 & 4 & 7 & 0 & 0 & 9 \\
\hline Patrik & SF & 9 & 2 & 2 & 2 & 0 & 1 & 3 \\
\hline Rosalinda & $\mathrm{N}$ & 2 & 3 & 7 & 2 & 2 & 3 & 7 \\
\hline Suzanna & $\mathrm{N}$ & 3 & 3 & 5 & 4 & 1 & 1 & 7 \\
\hline Tobby & $\mathrm{N}$ & 3 & 10 & 4 & 10 & 2 & 5 & 9 \\
\hline Tompong & $\mathrm{N}$ & 5 & 4 & 3 & 2 & 2 & 4 & 3 \\
\hline \multirow{2}{*}{ Totals } & & & 153 & 94 & 86 & 50 & 81 & 153 \\
\hline & & & \multicolumn{2}{|c|}{247} & \multicolumn{2}{|c|}{136} & \multicolumn{2}{|c|}{234} \\
\hline \multicolumn{3}{|l|}{ Unscorables } & \multicolumn{2}{|c|}{$\begin{array}{l}\text { (OMFs } \\
\text { discarded) }\end{array}$} & \multicolumn{2}{|c|}{111} & \multicolumn{2}{|c|}{13} \\
\hline
\end{tabular}




\section{Table 2 (on next page)}

Table of GLMM results 
2 Table 2. Optimal GLMM models for the effect of the factors facial orientation, recipient facial

3 expression and play intensity on the facial movement composition of OMF.

\begin{tabular}{lcccc}
\hline Predictor factors & Estimate & SE & Z & $\mathrm{p}$ \\
\hline Response factor: Number of & & & & \\
AUs & & & & \\
\hline Intercept & 0.68 & 0.15 & 4.55 & 0.000 \\
Facial orientation (FTF) & 0.36 & 0.14 & 2.59 & 0.009 \\
Recipient facial expression & 0.21 & 0.11 & 1.87 & 0.061 \\
(OMFR) & & & & \\
Play intensity (low) & 0.04 & 0.11 & 0.33 & 0.742 \\
\hline
\end{tabular}

Response factor: AU26 or AU27

\begin{tabular}{lcccc}
\hline Intercept & 0.095 & 0.51 & 0.19 & 0.852 \\
Facial orientation (FTF) & 1.65 & 0.69 & 2.38 & 0.017 \\
Recipient facial expression & 0.18 & 0.48 & 0.38 & 0.707
\end{tabular}

(OMFR)

$\begin{array}{lllll}\text { Play intensity (low) } & -0.61 & 0.50 & -1.23 & 0.219\end{array}$

\section{4}

